HIV

\title{
The effects of early syphilis on CD4 counts and HIV-1 RNA viral loads in blood and semen
}

\author{
S T Sadiq, J McSorley, A J Copas, J Bennett, S J Edwards, S Kaye, S Kirk, P French, IV D Weller
}

Sex Transm Infect 2005;81:380-385. doi: 10.1136/sti.2004.012914

See end of article for authors' affiliations .....................

Correspondence to: Dr Tariq Sadiq, Centre for Infection, Division of Cellular and Molecular Medicine, St George's University of London, Cranmer Terrace, London SW17 ORE, UK; ssadiq@ sgul.ac.uk

Accepted for publication 13 December 2004

\begin{abstract}
Objective: To examine the effect of early syphilis on blood and semen plasma HIV-1 viral loads and CD4 counts.

Methods: In a retrospective case-control study, blood plasma HIV-1 viral loads and CD4 counts in cases during early syphilis ( $n=63,27$ receiving antiretroviral therapy) were compared to those before and after syphilis and with controls with non-systemic acute sexually transmitted infections (STI) (n=104, 39 receiving antiretroviral therapy). In a prospective substudy in those not receiving antiretroviral therapy, semen plasma viral loads during early syphilis $(n=13)$ were compared with those 1 month, 3 months, and 6 months after treatment for syphilis and with controls with no STls $(n=20)$.

Results: Retrospective study: CD4 counts were similar in cases (median 410, $\mathrm{n}=139$ counts) during early syphilis compared to before $(485, n=80)$ and after $(475, n=88)$. In a secondary analysis, a drop in CD4 count $(21 \%)$ among those with early latent syphilis was observed compared with controls. Blood plasma viral loads did not change significantly overall or in those with primary, secondary, or early latent syphilis. Effects were similar on or off antiretroviral therapy. Prospective study: blood and semen viral loads were slightly higher in cases compared with controls but treatment of early syphilis did not reduce either.

Conclusions: We detected no association between early syphilis and changes in blood or semen viral load or CD4 count. Increased HIV-1 infectivity associated with early syphilis is unlikely to be associated with increased levels of HIV-1 RNA in blood or semen.
\end{abstract}

$\mathrm{T}$ he sexual transmission of syphilis and HIV-1 has been linked epidemiologically. ${ }^{1}$ In the United Kingdom, there has been an increase in the incidence of early syphilis affecting a substantial number of homosexual men with HIV1 infection. ${ }^{2}$ Syphilis has also been associated with increased replication of HIV-l in vitro, ${ }^{3}$ perhaps through its effects on immune activation and upregulation of chemokine coreceptors. ${ }^{4-11}$ Such effects, in those without syphilis, have been associated with increased HIV-1 RNA loads and decline in $\mathrm{CD} 4+\mathrm{T}$ cell concentration (CD4 count) in vivo ${ }^{12-16}$ and, when caused by systemic infections resolve, following treatment. ${ }^{17-19}$ In Africa, genito-ulcerative disease has been associated with increased shedding of HIV-1 from the female and male genital tracts. ${ }^{20-22}$ Thus, through these mechanisms early syphilis infection may result in increased infectivity of HIV- 1 and perhaps exacerbate immune depletion. In order to test this hypothesis we studied the effects of early syphilis on blood plasma viral loads (BPVL) and CD4 counts in a retrospective case control study. In a prospective substudy, we also examined effects of early syphilis on semen plasma HIV-1 RNA loads (SPVL).

\section{METHODS}

\section{Retrospective study}

All cases of early syphilis (primary, secondary, and early latent) in HIV-1 infected patients attending between January 2000 and September 2002 were identified from clinic and microbiological records. Standard diagnostic definitions were used for primary and secondary cases. ${ }^{23}$ The UK definition of early latent syphilis (those with positive syphilis serology combined with either a negative test for syphilis in the previous 2 years or a clinical picture compatible with infection with syphilis in that time) was used. For each case, controls were selected from among HIV-l infected men attending with a new episode of gonorrhoea, chlamydia, or non-specific urethritis. These sexually transmitted infections
(STI) were chosen because of evidence that they do not affect BPVL in men. ${ }^{24}$ Cases and controls with concurrent active genital herpes were excluded because of its effect of increasing BPVL. ${ }^{25}$ Controls were matched to cases by date of attendance and combination antiretroviral therapy (CART) status (that is, receiving or not receiving CART). All results of syphilis serology, dark ground examination of genital exudates, CD4 counts, and BPVLs taken while patients remained either on or off CART and within 1 year around the presentation of syphilis or STI were recorded. In those who had presented more than once with a new episode of syphilis in the period of study, the first diagnosis of syphilis was regarded as the index infection. Controls were only selected and used once.

For every case, a "period of syphilis disease" (POD) was defined (fig 1). A pre-POD and post-POD were also defined as immediately before and after the POD respectively, and equalling its duration. Controls had PODs assigned to them of the same duration as that in their matched case. The POD was intended to reflect a time period, wide enough to include the results of routine BPVLs and CD4 counts but narrow enough to include only times when HIV-1 replication may have been affected by syphilis. The POD included the estimated time that syphilis was incubating (primary $=30$ days, secondary $=90$ days, early latent $=120$ days), the period from the development of symptoms of syphilis to presentation to the clinic, the time from presentation to treatment, and an interval of 2 months after treatment (fig 1). This interval was chosen because of evidence that

Abbreviations: BPVL, blood plasma viral loads; CART, combination antiretroviral therapy; FACS, fluorescent activated cell sorter; GEE, generalised estimating equations; NSU, non-specific urethritis; PCR, polymerase chain reaction; $P O D$, period of syphilis disease; RPR, rapid plasma reagin; SPVL, semen plasma HIV-1 RNA loads; STI, sexually transmitted infections 


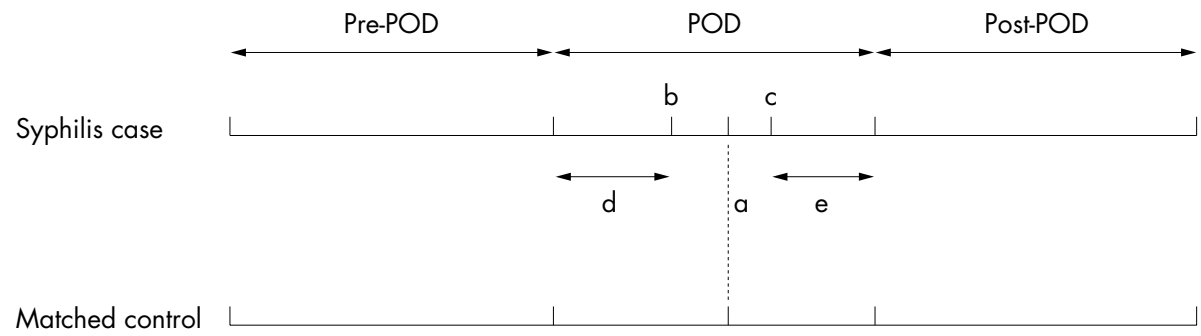

Figure 1 Schematic diagram representing definition of disease periods of syphilis and matched controls in the retrospective study. $a$, presentation to clinic with syphilis (for cases) or STI (for controls); $b$, beginning of symptoms/signs (for cases of early latent syphilis $b=a$ ); $c$, time of treatment of syphilis; $d$, incubation period of syphilis (primary $=30$ days, secondary $=90$ days, early latent $=120$ days); e, 2 months after treatment of syphilis; POD, period of syphilis disease; Pre-POD, before period of syphilis disease; Post-POD, after period of syphilis disease.

following treatment of acute systemic infections, such as bacterial pneumonia or malaria, BPVLs may remain elevated for 1 month $^{26}{ }^{27}$ returning to baseline usually by 3 months. $^{1418}$

As a secondary analysis, an additional period of disease, the "tight POD," was defined as a period from 2 weeks before to 2 weeks after presentation with syphilis in order to more readily "capture" the effect of syphilis. This shorter disease period was therefore likely to include fewer routine BPVL tests and CD4 counts.

Approximately 60 cases and 60 controls were required for $80 \%$ power if the average change in BPVL from non-POD to POD differed by $0.5 \mathrm{log}$, relative to a standard deviation of changes in each group of 1 , with a $5 \%$ significance level. To increase the power we attempted to select two controls for every case.

\section{Prospective substudy}

Recruitment to this study took place within the study period of the retrospective study-that is, from January to August 2002. HIV-1 infected men not on CART for at least 3 months, presenting either with untreated early syphilis (cases) or without syphilis and a negative STI screen on the day of attendance (controls) were recruited. This choice of controls without STIs was made because chlamydia, gonorrhoea, and non-specific urethritis (NSU) may affect HIV-l RNA levels in semen. ${ }^{24} 28$ Medical history, blood for syphilis serology, CD4 counts, and BPVL were taken and semen samples provided for assay of SPVL. Semen samples were provided after a routine urethral smear (testing for gonococcal, chlamydial, and non-specific urethritis) but before voiding urine. Standard treatment, usually a single dose of benzathine penicillin or a 2 week course of doxycyline, was given to those with syphilis. Patients were asked to return at 1 month, 3 months, and 6 months after treatment, providing blood and semen samples as previously for HIV-l RNA load measurement. Additionally, archived residues of BPVL samples routinely taken from participants before acquiring syphilis or presentation were retrieved for viral load analysis. Sixteen cases and 16 controls were required for $80 \%$ power if the average change in blood viral loads before and after treatment was 0.5 log compared to controls, relative to a standard deviation of changes within each group of 0.5 , and with a $5 \%$ significance level. The standard deviation was assumed to be lower than in the retrospective study because none of the patients were on CART and therefore possibly more homogeneous (as subsequently found in the results for viral load range; see table 1). We aimed to recruit 20 cases and 20 controls in case the standard deviation was somewhat higher than assumed.

\section{Testing of samples}

Semen and blood samples were centrifuged within 2 hours of collection and the plasma and cellular components stored at $-70^{\circ} \mathrm{C}$. HIV-1 RNA was extracted from blood and semen plasma by a silica gel capture method and quantified using a reverse transcribed polymerase chain reaction (PCR), clade B specific assay. ${ }^{30}{ }^{31} \mathrm{CD} 4$ counts were measured using fluorescent activated cell sorter (FACS) analysis. Treponema pallidum haemaglutination, rapid plasma reagin (RPR), and fluorescent treponemal assays were used to diagnose or exclude syphilis.

The studies were approved by the Camden and Islington Community Health Services local research ethics committee.

Table 1 Viral loads and CD4 counts of cases and controls in retrospective and prospective studies

\begin{tabular}{lll}
\hline & Syphilis & Controls \\
\hline Retrospective study (numbers) & 63 & 104 \\
Median pre-POD viral load (range) & $5500(<50-491000)$ & $3100(<50-938300)$ \\
Median POD viral load (range) & $16700(<50-3764500)$ & $1600(<50-6430700)$ \\
Median post-POD viral load (range) & $17400(<50-696900)$ & $1400(<50-730300)$ \\
Median pre-POD CD4 count (range) & $485(100-1180)$ & $460(64-1140)$ \\
Median POD CD4 count (range) & $410(90-1270)$ & 0.16 \\
Median post-POD CD4 count (range) & $475(120-1400)$ & $435(24-1160)$ \\
Prospective study (numbers) & 14 & $440(20-1140)$ \\
Median BPVL on available stored samples before syphilis† & $70359(2335-184084)$ & 20 \\
Median BPVL at first study visit & $36638(16998-287030)$ & $35578(2947-151652)$ \\
Median BPVL at study visits 2, 3, and 4 & $88786(17476-299401)$ & $4665(2519-80127)$ \\
Median SPVL at first study visit & $21060(<1,000-147531)$ & $3478(<1000-24171)$ \\
Median SPVL at study visits 2, 3, and 4 & $39412(<1000-196840)$ & 0.51 \\
\hline
\end{tabular}

POD, period of syphilis disease (see methods); BPVL, blood plasma viral load; SPVL, semen plasma viral load.

*Statistical tests based on comparison of mean log measures, using GEE where there are multiple measurements per patient, and $t$ test otherwise †Stored samples were available for analysis on 9 cases of syphilis and 9 controls. 
Statistical analysis

BPVLs and CD4 counts in retrospective study

BPVLs and CD4 counts in cases were compared to those in controls during the pre-POD, POD, and post-POD. Average changes in BPVL and CD4 count between different disease periods were then estimated separately for cases and controls. For primary analysis, changes were compared between cases and matched controls by testing for an interaction between disease period and whether case or control. All analysis was based on generalised estimating equations (GEE) of Stata 7, because of multiple measurements for patients and matching of controls, with exchangeable working correlation.

\section{BPVLs and SPVLs in prospective study}

BPVLs and SPVLs in cases were compared to those in controls at different study visits. Average changes in HIV-1 RNA loads across study visits were estimated separately for cases and controls, and then compared. For the change in HIV-1 RNA load, using multiple measurements for patients, analysis was based on GEE. When comparing HIV-1 RNA loads at particular times (for example, visit 1), the $t$ test was used.

\section{Secondary analysis}

Planned subgroup analysis included comparisons of latent with primary/secondary cases. Primary and secondary cases were grouped together because of the frequent difficulty in distinguishing these stages among those with concurrent HIV-1 infection. ${ }^{32}{ }^{33}$ Also planned for the retrospective study was the comparison between patients on and off CART. To determine whether changes in patient measures differed according to subgroup, the interaction was tested.

\section{Correlation of measures}

The Spearman rank correlation coefficient was calculated for semen and blood viral loads at visit 1 in the prospective study. In the retrospective study, to assess the correlation GEE was used to regress the changes in viral load on the corresponding changes in CD4 count.

\section{Outcome definition and summary statistics}

To provide simple summary statistics for CD4 and viral load we present the median and range; however, for all statistical testing as specified in our primary and secondary analyses we use the mean and the log (base 10) of the measurements. Undetectable viral load results were analysed as if half the detectability limit-for example, 25 copies $/ \mathrm{ml}$ in the retrospective study. Findings for CD4 count are presented as percentage change in CD4 count.

\section{RESULTS}

\section{Retrospective study}

In all, 63 cases of early syphilis were identified ( 15 primary syphilis, 37 secondary syphilis, and 11 early latent syphilis) and 104 controls (with gonorrhoea or non-gonococcal infection) were selected. All but two study participants were homosexual men; 27 cases and 39 controls were on CART. The median age, stage of HIV disease, and time since diagnosis of HIV were similar for cases and controls (data not shown). No patients in the study had a clinical and serological presentation compatible with primary HIV infection or seroconversion to HIV in the previous 3 months. Median BPVLs and CD4 counts before the time of having syphilis (the pre-POD) were similar in cases and controls. BPVLs during the defined syphilis period (the POD) and period after syphilis (the post-POD) were not significantly different in cases and controls (see table 1).

Overall, changes in BPVLs were not significant when comparing pre-POD to POD or POD to post-POD in all cases, in primary/secondary or early latent cases and in controls (fig 2) or when comparing cases to controls (data not shown). There were no significant associations between changes in BPVL and CART status. When using the tight POD, changes were broadly similar (data not shown).

Overall, no differences in CD4 counts were detected when comparing POD to pre-POD or post-POD in all cases or controls. Significant changes in CD4 counts through the PODs were detected in early latent cases (fig 2). When these changes where compared with changes in matched controls they remained significant, absolute CD4 counts decreasing relative to controls when moving from pre-POD to POD by $21 \%$ (95\% CI: $5 \%$ to $35 \%$ ) and then increasing relative to controls by a factor of $29 \%$ ( $7 \%$ to $55 \%$ ) following treatment for syphilis. The drop in CD4 counts in the POD, among early latent cases, was also significantly greater compared to primary and secondary cases, and this remained significant on comparison with matched controls (data not shown). Changes in CD4 count were not significantly related to changes in BPVL among cases or controls (data not shown).

\section{Prospective substudy}

A total of 13 cases with early syphilis (one with primary, seven with secondary, and five with early latent) and 20 controls were recruited. All except one case were also included in the main study.

The median time since HIV-1 diagnosis, stage of HIV infection, and age at entry into the study were similar in cases and controls (data not shown). All cases had positive

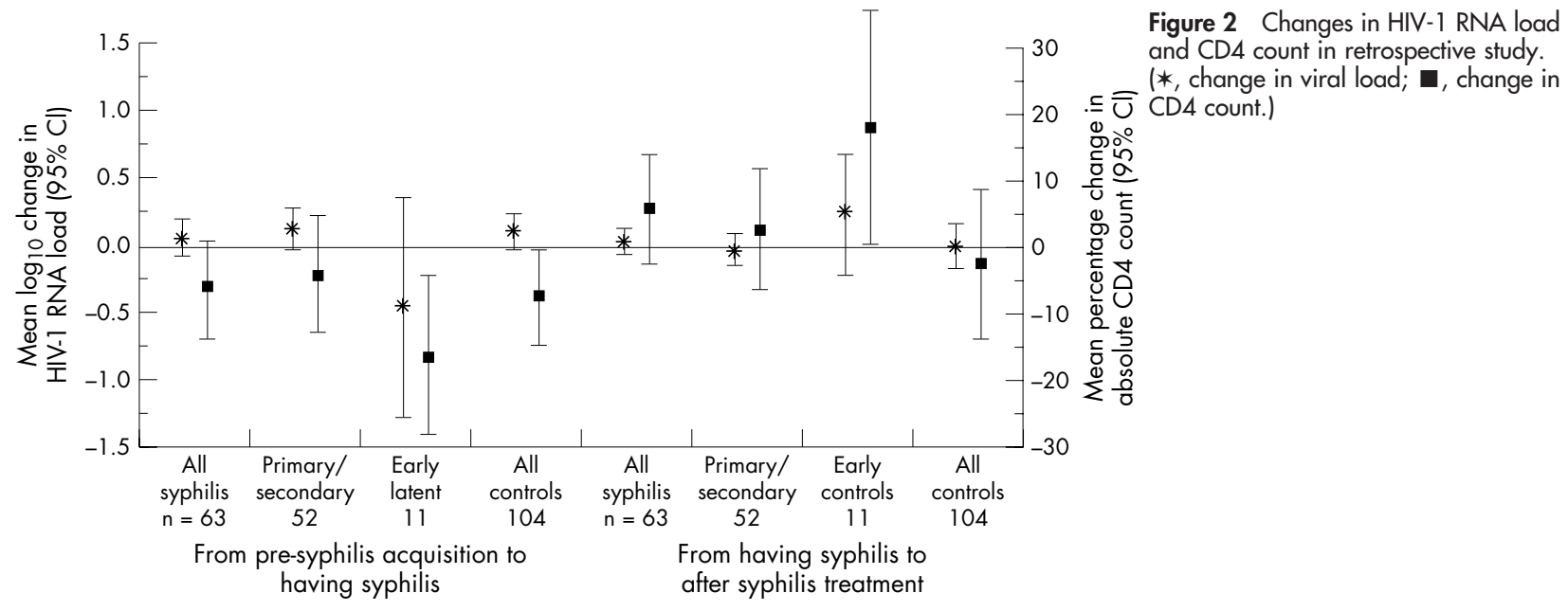




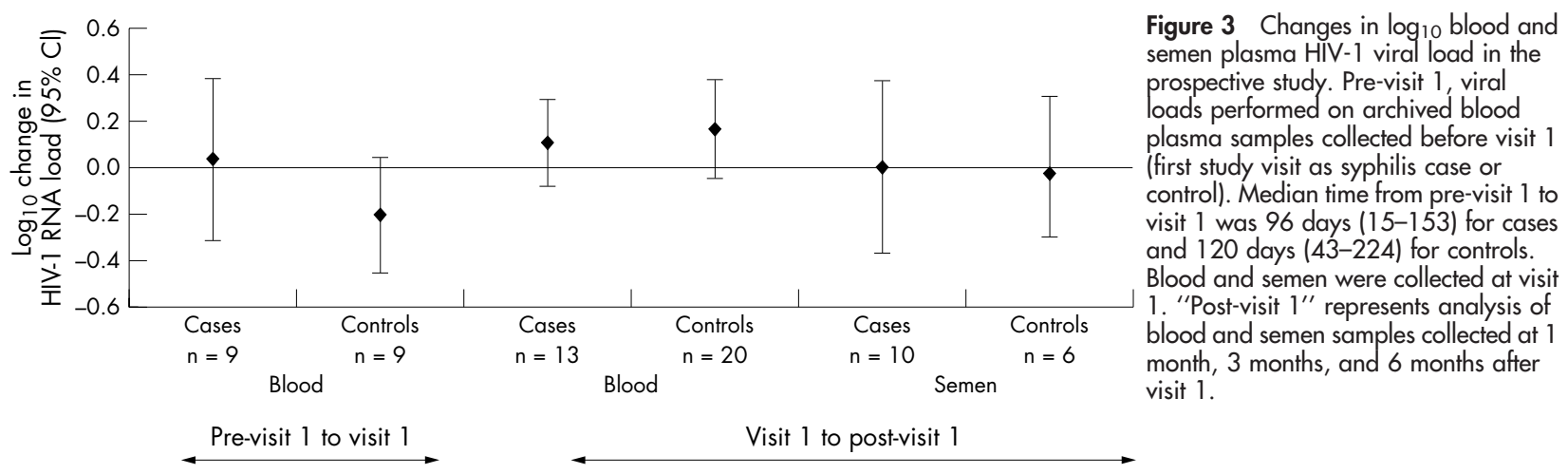

syphilis serology with RPR tests ranging from eight to 512 . Before the study and before syphilis no differences in BPVLs were detected between cases and controls (see table 1). Eleven cases and 19 controls gave semen as well as blood samples at the first study visit; 11 cases but only six controls were able to give semen samples at at least one follow up study visit. In nine cases and nine controls, archived blood plasma samples collected before infection with syphilis or first presentation (see methods) were retrieved. The median time between collection of these samples and first study visit was 96 days (15-153) for cases and 120 days (43-224) for controls ( $p=0.59$ for the difference, Mann-Whitney test). Overall, SPVLs correlated to BPVLs at visit l $(r=0.364$; $\mathrm{p}=0.048$; Spearman's rank coefficient).

BPVLs and SPVLs were higher among cases compared with controls at the first study visit (see table 1). At follow up visits SPVLs remained significantly higher, and there was some evidence that BPVLs also remained higher. No significant change in mean BPVL or SPVL was detected between visits in cases or controls and changes in cases were not significantly different from the changes in controls (fig 3). However, the confidence interval for the changes in cases relative to changes in controls from pre-syphilis to syphilis infection was relatively wide $(-0.17 \log -0.65 \log )$.

\section{DISCUSSION}

We studied the effects of early syphilis and its treatment on HIV-1 RNA loads in blood and on CD4 counts in a retrospective case control study and on HIV-1 RNA loads in semen in a small prospective substudy.

BPVLs were similar in cases and controls in the retrospective study, but both BPVLs and SPVLs were higher in cases compared with controls in the prospective study at presentation. Overall, however, the changes in HIV-1 RNA loads across disease periods among cases and relative to controls were not significant in both studies.

Generally, SPVLs tend to be lower than BPVLs (between 2fold and 10 -fold $)^{34}$ but correlate closely with them. Thus, the higher SPVLs observed in cases in the prospective study probably reflected the higher BPVLs of this group of patients. In the larger retrospective study, which included these cases in addition to many more, we deliberately chose controls presenting with non-systemic STIs and no differences in BPVL were observed between cases and controls, nor significant changes in BPVL among cases. Consequently, it is natural to suppose that in the prospective study the finding that BPVLs were higher in syphilis cases than controls may reflect the difference between the selection of controls in the two studies. In the prospective study controls were selected from those asymptomatic but deliberately seeking an STI check up (that was negative), as we wanted to measure viral loads in semen. Such controls may differ from cases in important respects leading to confounding for comparisons between cases and controls of BPVLs or SPVLs at given time periods, though less so for comparisons of the change in these measures. Although in this prospective study a relatively small number of patients were studied, the confidence limits around the mean values of blood and semen plasma viral loads make it unlikely that the average change, in general, is large. Interestingly, in a study among HIV-1 infected men with primary or secondary syphilis, without controls, BPVLs increased by a small $(0.21 \mathrm{log})$ but significant amount from pre-syphilis to syphilis infection. In those with secondary syphilis and those not on CART the rise was slightly higher $\left(0.33 \log\right.$ and $0.25 \log$ respectively).$^{35}$ In our retrospective study it is possible that CART may have limited the potential changes in BPVL through PODs, though in our subgroup analyses we found no significant evidence of differential change by CART status. Taking the two studies together the data suggest that if blood viral loads increase as a result of early syphilis the rises are small. The hypothesis that semen is an important source of increased HIV-1 infectivity during early syphilis is not strongly supported by the data from our prospective or retrospective studies. However, the possibility that semen viral loads increased because of syphilis and then failed to fall following penicillin therapy cannot be completely refuted and requires further investigation. It is likely that syphilitic chancres are a more important source of infective HIV-l as seen with genital herpes. ${ }^{36}$ Syphilitic lesions are infiltrated by CD4 T cells, histiocytes, and CD8+ cytotoxic T lymphocytes, thus providing a mechanism for transmission of both cell free and cell associated HIV-1..$^{6-8}$ Clearly, however, this remains an area for further research.

Overall, CD4 counts were similar in cases and controls in the retrospective study. However, in those with early latent syphilis, when compared with changes in controls, CD4 counts dropped significantly when compared to their prePOD and then increased following treatment of syphilis. This was a surprise finding as we expected smaller drops in CD4 count for these patients compared to those with primary and secondary syphilis, since immune activation may be less. In the study by Buchacz et $a l^{35}$ a drop in CD4 count was detected in those with secondary syphilis but the effects on those with early latent syphilis was not investigated. In our study any finding from subgroup analysis on 11 patients should be viewed with caution and research involving larger numbers may shed more light on this. Active syphilis has previously been associated with a reduction in CD4 percentage in both HIV-2 infected and uninfected patients respectively. ${ }^{37}$ Thus, the CD4 count changes we observed may simply reflect the effects of syphilis, acting independently of HIV-l infection.

Some features of the design of the study need to be taken into account when interpreting the data. The choice of 
controls with STIs in the retrospective study may have underestimated viral load effects because of a potential effect of these STIs on viral load. However, although previous work has shown that genital gonococcal infection can increase BPVL and reduce CD4 counts in female commercial sex workers in Africa, ${ }^{38}$ other work from Africa ${ }^{24}$ and a study from our clinic (unpublished data) have shown that it does not increase BPVL or reduce CD4 count. Additionally, POD definitions may have resulted in a dilution of the effect of syphilis. In the United Kingdom, early latent syphilis is diagnosed in those with positive syphilis serology combined with either a negative test for syphilis in the previous 2 years or a clinical picture compatible with infection with syphilis in that time. However, patients with concealed primary chancres or those presenting between the stages of primary and secondary disease may also erroneously be diagnosed with early latent syphilis. The choice of the incubation period of 120 days for early latent syphilis was a pragmatic one. Thus, in some of those with early latent syphilis, the incubation periods used may have been excessively short or long resulting in considerable overlap of syphilis disease state in pre-POD and POD. This would have resulted in reduced changes in BPVL and an underestimation of the effect on CD4 count. In those diagnosed with primary or secondary syphilis, the duration of PODs may have been too wide, again resulting in an underestimation of effect. However, the fact that no changes in viral load were seen, when comparing tight POD to post-POD, does not support this explanation.

Our data demonstrate that early syphilis has little, if any, effect on blood or semen plasma HIV-1 RNA loads and no overall effect on CD4 count. Early latent syphilis may be associated with lower CD4 counts. If early syphilis infection increases the risk of HIV transmission from men, then this study suggests that this effect is unlikely to be primarily the result of an increase in blood or semen plasma levels. The effect is more likely to be the result of increased shedding from associated genital skin lesions, as has been seen with genital herpes.

\section{ACKNOWLEDGEMENTS}

We thank the staff and patients in the Bloomsbury Clinic, Mortimer Market Centre. Martin Prince, senior MLSO, Microbiology, UCH, and Patrick Pearson, Centre for Sexual Health and HIV research for assistance in identifying cases of early syphilis.

\section{CONTRIBUTORS}

STS conceived the project and together with AJC, JM, PF, and IVDW designed it; STS and JM designed the database and together with SE collected and entered data; STS, JB, SK, and SK conducted the virological experiments; STS wrote the manuscript with major input from IVDW and AJC; all authors contributed to the writing the paper.

\section{Key messages}

- Early syphilis, whether primary, secondary or latent appears to have little effect on blood plasma HIV-1 RNA load either in those taking or not taking antiretroviral therapy

- Semen plasma HIV-1 RNA load does not fall following treatment of early syphilis

- Increased infectivity of HIV-1 associated with early syphilis is probably not the result of increased HIV-1 concentrations in semen but rather is associated with genital ulceration

- Early latent syphilis may be associated with depressed CD4 counts that are reversible on treatment of syphilis

\section{Authors' affiliations}

S T Sadiq, A J Copas, I V D Weller, Centre for Sexual Health and HIV Research, Department of Primary Care and Population Sciences, Royal Free and University College Medical School, University College, London, UK

J McSorley, S J Edwards, P French, Camden Primary Care Trust, London, UK

J Bennett, S Kaye, S Kirk, Centre for Virology, Division of Infection and Immunity, Royal Free and University College Medical School, University College, London, UK

S T Sadiq, Centre for Infection, Division of Cellular and Molecular Medicine, St George's University of London, London, UK

Source of funding: internal funding.

Conflicts of interest: none.

\section{REFERENCES}

1 Fleming DT, Wasserheit JN. From epidemiological synergy to public health policy and practice: the contribution of other sexually transmitted diseases to sexual transmission of HIV infection. Sex Transm Infect 1999;75:3-17.

2 Doherty L, Fenton KA, Jones J, et al. Syphilis: old problem, new strategy. BMJ 2002;325:153-6.

3 Theus SA, Harrich DA, Gaynor R, et al. Treponema pallidum, lipoproteins, and synthetic lipoprotein analogues induce human immunodeficiency virus type 1 gene expression in monocytes via NF-kappaB activation. J Infect Dis 1998; 177:941-50.

4 Tai KF, Ma Y, Weis JJ. Normal human B lymphocytes and mononuclear cells respond to the mitogenic and cytokine-stimulatory activities of Borrelia burgdorferi and its lipoprotein OspA. Infect Immun 1994;62:520-8.

5 Norgard MV, Arndt LL, Akins DR, et al. Activation of human monocytic cells by Treponema pallidum and Borrelia burgdorferi lipoproteins and synthetic lipopeptides proceeds via a pathway distinct from that of lipopolysaccharide but involves the transcriptional activator NF-kappa B. Infect Immun 1996;64:3845-52.

6 Tosca A, Lehou J, Hatjivasiliou M, et al. Infiltrate of syphilitic lesions before and after treatment. Genitourin Med 1988;64:289-93.

7 McBroom RL, Styles AR, Chiu MJ, et al. Secondary syphilis in persons infected with and not infected with HIV-1: a comparative immunohistologic study. Am J Dermatopathol 1999;21:432-41.

8 Engelkens $\mathrm{HJ}$, ten Kate FJ, Judanarso J, et al. The localisation of treponemes and characterisation of the inflammatory infiltrate in skin biopsies from patients with primary or secondary syphilis, or early infectious yaws. Genitourin Med 1993;69:102-7.

9 Van Voorhis WC, Barrett LK, Koelle DM, et al. Primary and secondary syphilis lesions contain mRNA for Th1 cytokines. J Infect Dis 1996;173:491-5.

10 Radolf JD, Arndt LL, Akins DR, et al. Treponema pallidum and Borrelia burgdorferi lipoproteins and synthetic lipopeptides activate monocytes/ macrophages. J Immunol 1995;154:2866-77.

11 Sellati TJ, Wilkinson DA, Sheffield JS, et al. Virulent Treponema pallidum lipoprotein, and synthetic lipopeptides induce CCR5 on human monocytes and enhance their susceptibility to infection by human immunodeficiency virus type 1. J Infect Dis 2000;181:283-93.

12 Reynes J, Portales P, Segondy M, et al. CD4+ T cell surface CCR5 density as a determining factor of virus load in persons infected with human immunodeficiency virus type 1. J Infect Dis 2000;181:927-32

13 Reynes J, Portales P, Segondy M, et al. CD4 T cell surface CCR5 density as a host factor in HIV-1 disease progression. AIDS 2001;15:1627-34.

14 Bush CE, Donovan RM, Markowitz NP, et al. A study of HIV RNA viral load in AIDS patients with bacterial pneumonia. J Acquir Immune Defic Syndr Hum Retrovirol 1996;13:23-6.

15 Leng Q, Borkow G, Weisman Z, et al. Immune activation correlates better than HIV plasma viral load with CD4 T-cell decline during HIV infection. J Acquir Immune Defic Syndr Hum Retrovirol 2001;27:389-97.

16 Wahl SM, Greenwell-Wild T, Peng G, et al. Mycobacterium avium complex augments macrophage HIV-1 production and increases CCR5 expression. Proc Natl Acad Sci USA 1998;95:12574-9.

17 Israel-Biet D, Cadranel J, Even P. Human immunodeficiency virus production by alveolar lymphocytes is increased during Pneumocystis carinii pneumonia. Am Rev Resp Dis 1993;148:1308-12.

18 Donovan RM, Bush CE, Markowitz NP, et al. Changes in virus load markers during AIDS-associated opportunistic diseases in human immunodeficiency virus-infected persons. J Infect Dis 1996;174:401-43

19 Denis M, Ghadirian E. Mycobacterium avium infection in HIV-1-infected subjects increases monokine secretion and is associated with enhanced viral load and diminished immune response to viral antigens. Clin Experiment Immunol 1994;97:76-82.

20 Lawn SD, Subbarao S, Wright TC Jr, et al. Correlation between human immunodeficiency virus type 1 RNA levels in the female genital tract and immune activation associated with ulceration of the cervix. $J$ Infect Dis 2000;181:1950-6.

21 Ghys PD, Fransen K, Diallo MO, et al. The associations between cervicovaginal HIV shedding, sexually transmitted diseases and immunosuppression in female sex workers in Abidjan, Cote d'lvoire. AIDS 1997; 11:F85-F93.

22 Dyer JR, Eron JJ, Hoffman IF, et al. Association of CD4 cell depletion and elevated blood and seminal plasma human immunodeficiency virus type 
(HIV-1) RNA concentrations with genital ulcer disease in HIV-1-infected men in Malawi. J Infect Dis 1998;177:224-27.

23 Holmes KK, Sparling PF, Mardh P, et al. Sexually tranmitted diseases, 3rd ed. New York: McGraw Hill, 1998.

24 Cohen MS, Hoffman IF, Royce RA, et al. Reduction of concentration of HIV-1 in semen after treatment of urethritis: implications for prevention of sexual transmission of HIV-1. AIDSCAP Malawi Research Group. Lancet 1997;349:1868-73

25 Mole L, Ripich S, Margolis D, et al. The impact of active herpes simplex virus infection on human immunodeficiency virus load. J Infect Dis 1997; 176:766-70.

26 Sulkowski MS, Chaisson RE, Karp CL, et al. The effect of acute infectious illnesses on plasma human immunodeficiency virus (HIV) type 1 load and the expression of serologic markers of immune activation among HIV-infected adults. J Infect Dis 1998;178:1642-8.

27 Hoffman IF, Jere CS, Taylor TE, et al. The effect of Plasmodium falciparum malaria on HIV-1 RNA blood plasma concentration. AIDS 1999; 13:487-494.

28 Eron JJ Jr, Gilliam B, Fiscus S, et al. HIV-1 shedding and chlamydial urethritis. JAMA 1996;275:36.

29 Sadiq ST, Taylor S, Copas A, et al. The effects of urethritis on seminal plasma HIV-1 RNA loads in homosexual men not receiving antiretroviral therapy. Sex Transm Infect 2005;81:120-3.

30 Boom R, Sol CJ, Salimans MM, et al. Rapid and simple method for purification of nucleic acids. J Clin Microbiol 1990;28:495-503.
31 Sadiq ST, Taylor S, Kaye S, et al. The effects of antiretroviral therapy on HIV-1 RNA loads in seminal plasma in HIV-positive patients with and without urethritis. AIDS 2002; 16:219-25.

32 Rompalo AM, Joesoef MR, O'Donnell JA, et al. Clinical manifestations of early syphilis by HIV status and gender: results of the syphilis and HIV study. Sex Transm Dis 2001;28:158-65.

33 Schofer H, Imhof M, Thoma-Greber E, et al. Active syphilis in HIV infection: a multicentre retrospective survey. The German AIDS Study Group (GASG) Genitourin Med 1996;72:176-81.

34 Tachet A, Dulioust E, Salmon D, et al. Detection and quantification of HIV-1 in semen: identification of a subpopulation of men at high potential risk of viral sexual transmission. AIDS 1999;13:823-31.

35 Buchacz K, Patel P, Taylor M, et al. Syphilis increases HIV viral load and decreases CD4 count cell counts in HIV-infected patients with new syphilis infections. AIDS 2004;18:2075-9.

36 Schacker T, Ryncarz AJ, Goddard J, et al. Frequent recovery of HIV-1 from genital herpes simplex virus lesions in HIV-1-infected men. JAMA 1998;280:61-6.

37 N'Gom PT, Jaffar S, Ricard D, et al. Immune stimulation by syphilis and malaria in HIV-2-infected and uninfected villagers in West Africa. Br J Biomed Sci 1997; 54:251-5

38 Anzala AO, Simonsen JN, Kimani J, et al. Acute sexually transmitted infections increase human immunodeficiency virus type 1 plasma viremia, increase plasma type 2 cytokines, and decrease CD4 cell counts. J Infect Dis $2000 ; 182: 459-66$.

\section{bmjupdates+}

bmjupdates+ is a unique and free alerting service, designed to keep you up to date with the medical literature that is truly important to your practice.

bmjupdates+ will alert you to important new research and will provide you with the best new evidence concerning important advances in health care, tailored to your medical interests and time demands.

Where does the information come from?

bmjupdates+ applies an expert critical appraisal filter to over 100 top medical journals A panel of over 2000 physicians find the few 'must read' studies for each area of clinical interest

Sign up to receive your tailored email alerts, searching access and more...

www.bmjupdates.com 